\title{
A escola como espaço para aprendizado sobre primeiros socorros
}

School as a place for learning first aid

La escuela como espacio de aprendizaje sobre primeros auxilios

\section{Monaliza Ribeiro Mariano Grimaldi ${ }^{1}$, Luci Maira Silva Gonçalves ${ }^{2}$, Ana Carolina de Oliveira Silva Melo ${ }^{3}$, Francisco Ivandi Melo ${ }^{4}$, Adriana Sousa Carvalho de Aguiar ${ }^{5}$, Morgama Mara Nogueira Lima ${ }^{6}$}

Resumo: Objetivos: implementar estratégia educativa sobre noções básicas de primeiros socorros com estudantes de escola pública e particular e verificar o conhecimento destes antes e após a intervenção educativa. Método: pesquisa quantitativa, descritiva, exploratória, realizada com 48 estudantes do nono ano. Para verificar o conhecimento, utilizaram-se dez questões objetivas e de múltipla escolha como pré e pós-teste. A coleta foi realizada de setembro a dezembro de 2016, totalizando seis encontros. Para análise utilizou-se Software PASW Statistics for Windows, versão 20.0, teste McNemar e Qui-quadrado. Resultados: nas escolas, houve diferença estatisticamente significativa entre os acertos no pós-teste sobre engasgo $\left(p^{2}=0,008\right)$, choque elétrico $\left(p^{2}=0,018\right)$, hemorragia $\left(\mathrm{p}^{2}=0,004\right)$, parada cardiorrespiratória $\left(\mathrm{p}^{2}=0,041\right)$. Logo, a média de acertos na escola pública e privada foi, respectivamente, $70,0 \%$ e $85,5 \%$. Conclusão: os estudantes obtiveram aprendizado significativo mediante intervenção educativa, ressaltando a relevância na inclusão da disciplina de primeiros socorros nas escolas.

Descritores: Promoção da saúde; Enfermagem; Adolescente; Educação em saúde

\footnotetext{
${ }^{1}$ Enfermeira. Doutora em Enfermagem. Universidade da Integração Internacional da Lusofonia Afro-Brasileira. Acarape, Ceará, Brasil. Email:monalizamariano@unilab.edu.br ORCID: http://orcid.org/0000-0002-8718-4783

${ }^{2}$ Enfermeira. Universidade da Integração Internacional da Lusofonia Afro-Brasileira. Acarape, Ceará, Brasil. E-mail: lucigonalves@gmail.com ORCID: http://orcid.org/0000-0002-7669-4593

${ }^{3}$ Enfermeira. Residente de enfermagem em terapia intensiva. Secretaria de Saúde do Estado do Ceará. Fortaleza, Ceará, Brasil. E-mail: carolina_enfermagem2@hotmail.com ORCID: http://orcid.org/0000-0002-2098-2545

${ }^{4} 41^{\text {}}$ Sargento do Corpo de Bombeiros Militar do Estado do Ceará. Fortaleza, Ceará, Brasil. E-mail: firemelllo@hotmail.com ORCID: http://orcid.org/0000-0003-1153-5759

${ }^{5}$ Enfermeira. Doutoranda do Programa de Pós-Graduação em Cuidados Clínicos em Enfermagem e Saúde. Universidade Estadual do Ceará. Fortaleza, Ceará, Brasil. E-mail: adrianaufc@gmail.com ORCID: http://orcid.org/0000-0002-2726-8707

${ }^{6}$ Enfermeira. Mestre em Enfermagem. Universidade da Integração Internacional da Lusofonia Afro-Brasileira. Acarape, Ceará, Brasil. E-mail: morgamamara@gmail.com ORCID: http://orcid.org/0000-0003-1012-0738
} 
A escola como espaço para aprendizado sobre primeiros socorros $\mid 2$

\begin{abstract}
Objectives: to implement an educational strategy about first aid in students from public and private schools and to verify their knowledge before and after the educational intervention. Method: quantitative, descriptive, exploratory research, carried out with 48 ninth-year students. In order to verify students' knowledge, ten objective and multiple choice questions were used pre and post-test. The collection was carried out from September to December 2016, totaling six meetings. Data analysis was carried out using PASW Statistics for Windows software, version 20.0. McNemar and Chi-square tests were used. Results: there was a statistically significant difference between the correct answers in the post-test questions about choking $\left(\mathrm{p}^{2}=0.008\right)$, electric shock $\left(\mathrm{p}^{2}=0.018\right)$, hemorrhage $\left(\mathrm{p}^{2}=0.004\right)$, and cardiorespiratory arrest $\left(\mathrm{p}^{2}=0.041\right)$. The average of correct answers in public and private schools were, respectively, $70.0 \%$ and $85.5 \%$. Conclusion: students obtained significant learning through the educational intervention, emphasizing the relevance of including the discipline of first aid in schools.
\end{abstract}

Descriptors: Health promotion; Nursing; Adolescent; Health education

Resumen: Objetivos: implementar una estrategia educativa sobre los conceptos básicos de primeros auxilios con estudiantes de escuelas públicas y privadas y verificar sus conocimientos antes y después de la intervención educativa. Método: investigación exploratória, cuantitativa, descriptiva, realizada con 48 alumnos del noveno año. Para verificar el conocimiento, se utilizaron diez preguntas objetivas y de opción múltiple como pre-test y post-test. La colección se llevó a cabo de septiembre a diciembre de 2016, totalizando seis reuniones. Para el análisis, se utilizó el software PASW Statistics para Windows, versión 20.0 y las pruebas de McNemar y Chi-cuadrado. Resultados: hubo una diferencia estadísticamente significativa entre las respuestas correctas en el post-test acerca de asfixia $\left(\mathrm{p}^{2}=0.008\right)$, descarga eléctrica $\left(\mathrm{p}^{2}=0.018\right)$, hemorragia $\left(\mathrm{p}^{2}=0.004\right)$ y paro cardiorrespiratorio $\left(\mathrm{p}^{2}=0.041\right)$. El número promedio de respuestas correctas en las escuelas públicas y privadas fue, respectivamente, $70.0 \%$ y $85.5 \%$. Conclusión: los estudiantes obtuvieron un aprendizaje significativo a través de la intervención educativa, enfatizando la relevancia de incluir la disciplina de primeros auxilios en las escuelas.

Descriptores: Promoción de la salud; Enfermería; Adolescente Educacion en salud

\title{
Introdução
}

Os acidentes, atualmente, são responsáveis por alto número de atendimentos nas urgências e emergências dos hospitais. Acidente é definido como um acontecimento fortuito, independente da vontade humana, provocado por uma força externa que age rapidamente, manifestando-se por um dano corporal ou mental, sendo um evento não esperado e não planejado, que mostra uma disfunção do meio onde ocorre. ${ }^{1}$

Acidentes de carro, atropelamentos, afogamentos, incêndios e acidentes no ambiente de trabalho ou domiciliar, como queimaduras, choque elétrico, quedas, intoxicação, ferimentos por objetos perfurocortantes, entre outros, são muito comuns, podendo ocorrer a qualquer momento, 
lugar e ocasião. Entretanto, geralmente, ocorrem em ambiente domiciliar; assim, os familiares e as próprias vítimas são elementares a prestar os primeiros socorros ou tomar as primeiras atitudes no momento do acidente. ${ }^{2}$ Ainda, os primeiros socorros, também, podem ser utilizados para pacientes com doenças crônicas agudizadas, doenças clínicas, em que o paciente precisa de intervenção imediata para que não haja complicações.

Os primeiros socorros são os procedimentos iniciais de emergência, de menor complexidade, visando preservar a vida e evitar danos maiores até que a pessoa receba assistência especializada. Esse atendimento inicial se baseia na assistência imediata à pessoa em uma situação de agravo à saúde, incluindo procedimentos que exijam ou não a mínima utilização de materiais ou equipamentos. Os objetivos desse atendimento são: preservar a vida, prevenir sequelas ou deterioração do estado de saúde da vítima e promover a recuperação, até que os profissionais da saúde assumam o atendimento, sempre fundamentado no princípio de não causar malefícios à vítima. ${ }^{3}$

Os procedimentos realizados são ações adequadas, que incluem o conhecimento, expressas nas respostas dadas pelos indivíduos envolvidos em situações que requerem ações imediatas. ${ }^{2}$ Certos procedimentos básicos de primeiros socorros poderão ser executados por qualquer pessoa que esteja junto à vítima. Outros procedimentos mais complexos deverão ser seguidos exclusivamente pela equipe médica, com a assistência da equipe de enfermagem e pessoas habilitadas para tal. ${ }^{4}$

A atuação do profissional de saúde, incluindo enfermeiro e socorristas, na prevenção desses agravos é pela manutenção de vida até a chegada do suporte básico ou avançado de vida e um transporte adequado a um serviço de saúde devidamente hierarquizado e integrado ao Sistema Único de Saúde. ${ }^{5}$ A realização do primeiro atendimento evita possíveis complicações, como por exemplo: agravamento do quadro clínico, lesões e até mesmo o óbito. 
A escola como espaço para aprendizado sobre primeiros socorros $\mid 4$

Em diversas situações de emergência, o leigo também pode rapidamente reconhecer o fato e iniciar o procedimento de cuidados emergenciais. Dentre estes, a pronta ativação do serviço especializado de emergência faz dele uma pessoa de fundamental importância na sobrevivência da vítima. O aumento da sobrevida, principalmente, no ambiente extra-hospitalar, está relacionado ao desempenho dos socorristas leigos. Entende-se por socorrista leigo a pessoa sem formação profissional na área da saúde e que pode intervir em uma situação de emergência, contribuindo com o aumento da sobrevivência e redução das sequelas da vítima. ${ }^{3}$

A orientação e capacitação da população em relação a situações de emergência são extremamente necessárias, devendo ser mais difundidas em ambientes coletivos, como escolas, empresas, academias e parques. Isso é verificado no protocolo de parada cardiorrespiratória para leigos, no qual se verificou que há ganho da sobrevida do paciente. ${ }^{6}$

A escola é um espaço onde crianças e adolescentes passam um período significativo do dia, sendo comum a ocorrência de acidentes por existirem locais de risco, dentre os quais se destacam: pátios, corredores, parques, banheiros, salas de aula, escadas e quadras poliesportivas. Assim, estão sujeitos a intercorrências, tornando-se importante o conhecimento adequado acerca das intervenções que devem ser realizadas em casos de situações emergenciais, pois um atendimento de emergência malfeito pode comprometer ainda mais a saúde da vítima.

Com isso, observa-se a importância de capacitar professores e funcionários dos estabelecimentos de ensino público e/ou privado de nível básico, bem como nos estabelecimentos de recreação infantil. O que pode ser observado na Lei 13.722, de 2018, que torna obrigatória a capacitação em noções básicas de primeiros socorros para os educadores e funcionários no âmbito educacional. ${ }^{7}$

Nas escolas, os estudantes também são importantes multiplicadores, repassando o conhecimento aos outros colegas, funcionários e familiares. Nesse contexto, as escolas têm um 
5 | Grimaldi MRM, Gonçalves LMS, Melo ACOS, Melo FI, Aguiar ASC, Lima MMN

papel importante e crescente na promoção de saúde, prevenção de doenças e de acidentes entre crianças e adolescentes. ${ }^{8} \mathrm{O}$ enfermeiro, como profissional educador, pode treinar estudantes para atuarem em situações de emergência.

Isso reforça a importância da realização de estratégias educativas sobre temática de primeiros socorros no âmbito escolar, pois ainda se percebe um deficit de informações relacionados a esse tema, o que torna importante a realização desse estudo no âmbito escolar. Além disso, de acordo com a literatura, poucos estudos são realizados com ênfase nessa temática com os estudantes. ${ }^{7}$

A partir dessa problemática identificou-se a necessidade de investigar as seguintes questões: Qual o conhecimento dos alunos acerca de noções básicas de primeiros socorros? A realização de estratégias educativas sobre essa temática na escola contribui para a aprendizagem deles?

Esta pesquisa teve como objetivos implementar estratégia educativa sobre noções básicas de primeiros socorros com alunos de escola pública e particular, bem como verificar o conhecimento deles antes e após a intervenção educativa.

\section{Método}

Estudo exploratório, descritivo, quantitativo, realizado em duas escolas, uma na cidade de Acarape e a outra em Fortaleza, ambas do estado do Ceará, sendo uma pública da rede municipal e a outra da rede privada, no período de setembro a dezembro de 2016.

Participaram do estudo alunos regularmente matriculados no turno diurno do nono ano do ensino fundamental. Foram excluídos estudantes que faltaram a algum dos encontros da estratégia educativa, sendo 14 de Fortaleza e 8 de Acarape. Do total de 70 (38 em Fortaleza e 32 em Acarape), participaram 48 estudantes, sendo 24 da escola pública e 24 da escola particular. A escolha do nono ano foi intencional, por contemplar estudantes com idade em torno de 15 anos 
A escola como espaço para aprendizado sobre primeiros socorros $\mid 6$

e por entender que eles teriam facilidade tanto de compreensão quanto de atenção do método que seria utilizado. Todos os alunos, 70 estudantes, desse ano do período diurno foram convidados a participar, porém 13 (8 da escola privada e 5 da escola pública) não entregaram a autorização dos pais e 9 faltaram a algum dos encontros (6 da escola privada e 3 da escola pública).

Antes de iniciar a coleta foi confirmado com o coordenador das escolas e professores do nono ano que esses alunos não tinham realizado nenhum treinamento prévio sobre primeiros socorros, para que não houvesse viés nos resultados.

A coleta dos dados, previamente agendada na escola, ocorreu em seis encontros. No primeiro encontro foram esclarecidos aos estudantes, de forma verbal, os objetivos e a importância da pesquisa e obtido seu consentimento e de seus responsáveis.

No segundo encontro, para avaliação do conhecimento prévio dos estudantes, foi aplicado um instrumento pré-teste elaborado mediante leitura e análise prévia de bibliografias sobre suporte básico de vida. Composto por dez questões objetivas de múltipla escolha, o instrumento de coleta de dados abordou condutas de primeiros socorros que devem ser tomadas nas principais situações de emergência. As questões versaram sobre temas como: $\mathrm{Q}_{1}$ - engasgo, $\mathrm{Q}_{2}-$ convulsão, $\mathrm{Q}_{3}-$ desmaio, $\mathrm{Q}_{4}-$ queimadura, $\mathrm{Q}_{5}$ - choque elétrico, $\mathrm{Q}_{6} \mathrm{e} \mathrm{Q}_{7}-$ hemorragia, $\mathrm{Q}_{8}-$ intoxicação, $\mathrm{Q}_{9}$ e $\mathrm{Q}_{10}$ - parada cardiorrespiratória.

A partir do terceiro encontro, aconteceram as oficinas sobre noções básicas de primeiros socorros nas principais situações de emergência, que totalizaram quatro encontros com duração de uma hora cada. As atividades educativas foram desenvolvidas por monitores graduandos em enfermagem e enfermeiras colaboradoras, que passaram por treinamento com profissional do Serviço de Atendimento Móvel de Urgência (Samu). 
Para a realização das oficinas, como estratégias educativas, utilizaram-se aulas expositivas, práticas e vídeos, que abordaram assuntos sobre condutas de primeiros socorros em situações de queimadura, choque elétrico, engasgo, desmaio, convulsão, hemorragias, intoxicação/envenenamento e parada cardiorrespiratória. Ressalta-se que as noções de biossegurança foram mencionadas, explicando a importância delas para segurança daqueles que prestam os primeiros socorros, a fim de que evitem contaminação ou que possam se tornar vítimas.

Após a abordagem do conteúdo teórico, os escolares participaram de uma demonstração prática imediata de todas as manobras em manequins simuladores. Ao término das quatro oficinas, foi aplicado o instrumento pós-teste com as mesmas questões do instrumento préteste, para verificar a assimilação da aquisição de informações pelos escolares acerca da temática abordada e avaliar o percentual de erros e acertos das questões.

Os dados foram processados no Software PASW Statistics for Windows (SPSS) versão 20.0, licença número 10101131007. Verificou-se a comparação dos acertos das questões antes e após a estratégia educativa para cada escola pelo Teste de McNemar. Também se analisou a comparação dos acertos das questões após a estratégia educativa entre as duas escolas pelo Teste de Qui-Quadrado, para avaliar qual escola obteve mais acertos. Consideraram-se como estatisticamente significantes as análises com $\mathrm{p}<0,05$.

O estudo foi aprovado pelo Comitê de Ética em Pesquisa com CAAE no 56673216.5.0000.5576 e respeitou os princípios éticos conforme Resolução 466/12. Pais e responsáveis assinaram o Termo de Consentimento Livre e Esclarecido e os escolares, o Termo de Assentimento Livre e Esclarecido.

\section{Resultados}


A escola como espaço para aprendizado sobre primeiros socorros $\mid 8$

Participaram 48 estudantes. A idade variou de 13 a 17 anos, sendo a maioria com 13 e 14 $\operatorname{anos}(66,6 \%$ e 75,0\%, respectivamente). Em relação ao sexo, houve predominância do sexo feminino nas duas escolas, particular $(70,8 \%)$ e pública $(53,3 \%)$.

Observa-se na tabela 1 que em ambas as escolas, de forma geral, os estudantes tiveram um alto percentual de acertos das questões após a intervenção educativa, demonstrando um bom aprendizado.

Tabela 1 - Relação do percentual de acertos das questões antes e depois (pré e pós-teste) da intervenção educativa nas escolas pública e privada. Acarape-CE, 2018.

\begin{tabular}{|c|c|c|c|c|c|c|c|c|c|c|c|}
\hline \multicolumn{6}{|c|}{ Pública } & \multicolumn{6}{|c|}{ Privada } \\
\hline & \multicolumn{2}{|c|}{ Antes } & \multicolumn{2}{|c|}{ Depois } & \multirow[t]{2}{*}{$\mathbf{P}^{1}$} & \multicolumn{2}{|c|}{ Antes } & \multicolumn{2}{|c|}{ Depois } & \multirow[t]{2}{*}{$\mathbf{P}^{1}$} & \multirow[t]{2}{*}{$\mathbf{P}^{2}$} \\
\hline & $\mathbf{N}$ & $\%$ & $\mathbf{N}$ & $\%$ & & $\mathbf{N}$ & $\%$ & $\mathbf{N}$ & $\%$ & & \\
\hline Q1 - Engasgo & 14 & 58,3 & 14 & 58,3 & 1,000 & 20 & 83,3 & 22 & 91,7 & 0,500 & 0,008 \\
\hline Q2 - Convulsão & 3 & 12,5 & 24 & 100,0 & 0,000 & 14 & 58,3 & 22 & 91,7 & 0,039 & $0,149^{*}$ \\
\hline Q3 - Desmaio & 13 & 54,2 & 21 & 87,5 & 0,021 & 13 & 54,2 & 20 & 83,3 & 0,039 & 0,683 \\
\hline Q4 - Queimadura & 17 & 70,8 & 22 & 91,7 & 0,125 & 19 & 79,2 & 22 & 91,7 & 0,250 & 1,000 \\
\hline $\begin{array}{l}\text { Q5 - Choque } \\
\text { elétrico }\end{array}$ & 21 & 87,5 & 19 & 79,2 & 0,687 & 20 & 83,3 & 24 & 100,0 & 0,125 & 0,018 \\
\hline Q6 - Hemorragia & 17 & 70,8 & 6 & 25,0 & $\mathbf{0 , 0 1 3}$ & 15 & 62,5 & 16 & 66,7 & 1,000 & 0,004 \\
\hline Q7 - Hemorragia & 8 & 33,3 & 10 & 41,7 & 0,727 & 4 & 16,7 & 16 & 62,5 & $\mathbf{0 , 0 0 1}$ & 0,149 \\
\hline Q8 - Intoxicação & 8 & 33,3 & 18 & 75,0 & 0,006 & 5 & 20,8 & 20 & 83,3 & $\mathbf{0 , 0 0 0}$ & 0,477 \\
\hline $\begin{array}{l}\text { Q9 - Parada } \\
\text { Cardiorespiratória }\end{array}$ & 14 & 58,3 & 18 & 75,0 & 0,180 & 11 & 45,8 & 22 & 91,7 & $\mathbf{0 , 0 0 0}$ & 0,081 \\
\hline Q10 - Parada & 9 & 37,5 & 16 & 66,7 & 0,012 & 4 & 16,7 & 22 & 91,7 & 0,000 & 0,041 \\
\hline
\end{tabular}

\section{Cardiorespiratória}

$\mathrm{p}^{1}$ : Teste de McNemar para comparação dos acertos das questões antes e depois da intervenção educativa em cada escola. p : Teste de Qui-Quadrado para comparação dos acertos das questões depois da intervenção entre as duas escolas. *Teste de razão de verossimilhança

Conforme a tabela 1, no pré-teste as questões com os percentuais mais baixos (inferior a $50 \%)$ de acertos na escola pública foram equivalentes às questões $\mathrm{Q}_{2}(12,5 \%), \mathrm{Q}_{7}(33,3 \%), \mathrm{Q}_{8}(33,3 \%)$ e $\mathrm{Q}_{10}(37,5 \%)$. De modo semelhante, na escola particular, foram as questões $\mathrm{Q}_{7}(16,7 \%), \mathrm{Q}_{8}(20,8 \%)$, $\mathrm{Q}_{9}(45,8 \%)$ e $\mathrm{Q}_{10}(16,7 \%)$. Portanto, os assuntos que os estudantes mais demonstraram desconhecimento foram sobre condutas de primeiros socorros em situações de convulsão, hemorragia, intoxicação e parada cardiorrespiratória. 
No pós-teste, as questões com mais de $80 \%$ de acertos entre os estudantes da escola-pública foram sobre os temas convulsão, queimadura e desmaio, respectivamente: $\mathrm{Q}_{2}(100,0 \%), \mathrm{Q}_{4}(91,7 \%)$ e $\mathrm{Q}_{3}(87,5 \%)$. Na escola particular, todas as questões no pós-teste tiveram índices de acertos acima de $80 \%$, exceto questões relativas à temática hemorragia: $\mathrm{Q}_{6}(66,7 \%)$ e $\mathrm{Q}_{7}(62,5 \%)$.

Ainda, percebe-se que houve uma diferença estatisticamente significante entre o pré e pósteste para as questões $\mathrm{Q}_{2}\left(\mathrm{p}^{1}=0,000\right), \mathrm{Q}_{3}\left(\mathrm{p}^{1}=0,021\right), \mathrm{Q}_{6}\left(\mathrm{p}^{1}=0,013\right), \mathrm{Q}_{8}\left(\mathrm{p}^{1}=0,006\right)$ e $\mathrm{Q}_{10}\left(\mathrm{p}^{1}=0,012\right)$, na escola pública. Entre os estudantes da escola particular, observou essa associação para as questões $\mathrm{Q}_{2}\left(\mathrm{p}^{1}=0,039\right), \mathrm{Q}_{3}\left(\mathrm{p}^{1}=0,039\right), \mathrm{Q}_{7}\left(\mathrm{p}^{1}=0,001\right), \mathrm{Q}_{8}\left(\mathrm{p}^{1}=0,000\right), \mathrm{Q}_{9}\left(\mathrm{p}^{1}=0,000\right)$ e $\mathrm{Q}_{10}\left(\mathrm{p}^{1}=0,000\right)$. Isso demonstra que as oficinas educativas contribuíram para o aumento do conhecimento em relação às condutas de primeiros socorros, principalmente, em situações de convulsão, desmaio, hemorragia, intoxicação e parada cardiorrespiratória.

Ainda na tabela 1 , os valores de $\mathrm{p}^{2}$ mostram a comparação dos acertos das questões após a intervenção educativa entre as duas escolas. Constatou-se diferença estatisticamente significante entre a escola pública e a privada com relação aos acertos no pós-teste para as questões que versavam sobre primeiros socorros em situações de engasgo, choque elétrico, hemorragia, parada cardiorrespiratória, respectivamente: $\mathrm{Q}_{1}\left(\mathrm{p}^{2}=0,008\right), \mathrm{Q}_{5}\left(\mathrm{p}^{2}=0,018\right), \mathrm{Q}_{6}\left(\mathrm{p}^{2}=0,004\right)$ e $\mathrm{Q}_{10}\left(\mathrm{p}^{2}=0,041\right)$.

No pós-teste a média do percentual de acertos das questões na escola pública foi de 70,0\% e na escola particular foi de $85,5 \%$. Observa-se que, apesar de o percentual de acertos ter sido maior na escola particular do que entre os estudantes da escola pública, em ambas as escolas houve um acréscimo de acertos no pós-teste em relação ao pré-teste, demonstrando que os estudantes foram capazes de agregar a maior parte do conteúdo discutido durante as oficinas.

\section{Discussão}

Compreendendo a escola como ambiente favorável para a formação de cidadãos, entende-se a necessidade de se trabalharem temáticas relativas à preservação da segurança humana, relacionada 
com a saúde, no que tange aos primeiros socorros. Estudo realizado com leigos, no qual estes desempenharam atividades educativas sobre prevenção de acidentes e primeiros socorros, possibilitou aos participantes o conhecimento e a atualização sobre a temática, favorecendo a eles serem multiplicadores dos conhecimentos adquiridos. ${ }^{9}$ Isso reforça a importância de as pessoas que se deparam com situações de emergência terem conhecimentos básicos de condutas adequadas.

Estudo realizado com 46 crianças de 9 a 13 anos utilizou duas estratégias diferentes para identificação de procedimentos sobre primeiros socorros, uma por meio de recurso lúdico e a outra somente aula expositiva. Em ambas foram empregados pré e pós-teste, verificando que a turma na qual foi aplicada a atividade lúdica obteve maior índice (87\%) de acertos nas questões que a turma com a aula tradicional (37\%). ${ }^{10} \mathrm{~A}$ utilização de recurso educativo contribui de forma efetiva como facilitador para o ensino-aprendizagem na escola.

Os resultados deste estudo apontaram, em relação ao conhecimento, melhora significativa imediatamente após as oficinas educativas. Constatou-se que em ambas as escolas houve um aumento nos acertos das questões no pós-teste comparado com o pré-teste, mostrando assim que há capacidade de aquisição de conhecimentos teóricos e práticos, associada à sua aplicabilidade em situações potenciais que podem ser vivenciadas no ambiente escolar.

Em estudo que objetivou avaliar o conhecimento de professores e funcionários de uma escola, por meio de abordagens teóricas e práticas, observou-se aumento significativo da porcentagem de acertos após o treinamento em primeiros socorros. Verificou-se, antes do treinamento, uma pontuação média de 19,43 pontos referentes a habilidade e 2,91 pontos no conhecimento e, após o treinamento, 174,57 pontos na habilidade e 9,17 no conhecimento, diferença estatisticamente significativa $(\mathrm{p}<0,001)$. Constatou-se um aumento significativo da porcentagem de acertos após o treinamento em primeiros socorros. ${ }^{8}$ Ainda, em estudo com 110 
11 | Grimaldi MRM, Gonçalves LMS, Melo ACOS, Melo FI, Aguiar ASC, Lima MMN

professores pré-escolares, verificou-se conhecimento prévio insuficiente em práticas básicas de primeiros socorros. A pontuação média dos conhecimentos foi de $11,9 \pm 2,9$, para 20 pontos. ${ }^{11}$

As circunstâncias que requerem atenção médica são comuns nas escolas e os professores são muitas vezes os primeiros a presenciar situações de emergência com os estudantes. Isso reforça a importância do papel que o ambiente educacional desempenha na promoção de saúde e prevenção de doenças e acidentes, da mesma forma que é fundamental que existam pessoas capacitadas para oferecer esse suporte no âmbito escolar, como os profissionais da saúde. ${ }^{12}$

Esses treinamentos devem ser permanentes, nos quais os conteúdos sejam apresentados visualmente com demonstrações práticas, simulação de atendimentos e construção de cenários próximos das situações reais. Essas metodologias favorecem a aquisição não só de conhecimentos teóricos, mas também de habilidades. ${ }^{13}$

Neste estudo, observou-se, pelo percentual de acertos antes das oficinas educativas, que os alunos trazem consigo alguns conhecimentos construídos do senso comum, adquiridos por meio da interação com contexto social. Estratégias educativas sobre prevenção de acidentes e primeiros socorros no ambiente escolar são fundamentais para ampliação desse conhecimento.

O ensino de primeiros socorros deveria ser amplamente disponibilizado e democratizado, não ficar restrito aos profissionais de saúde ou àqueles de universidades e hospitais. Confere aos usuários maior segurança para tratar de seus problemas de saúde, reduzindo sua vulnerabilidade. ${ }^{14}$

Existem estratégias que favorecem o ensino de "primeiros socorros" na escola, como o Programa de Saúde na Escola (PSE) e o projeto Samuzinho. O PSE, desde 2007, estimula a intersetorialidade entre saúde e educação devido à atuação da Estratégia de Saúde da Família (ESF). ${ }^{15}$ O projeto Samuzinho foi idealizado em 2007, pelo Serviço Atendimento Médico de Urgência, do Distrito Federal, com o objetivo de conscientizar, e também capacitar, as crianças em relação aos problemas causados por ligações indevidas ao número de emergência $192 .{ }^{16}$ Essas 
estratégias sobre a temática "primeiros socorros" na escola contribuem com as atividades de educação em saúde, tanto para professores quanto para alunos. ${ }^{17}$

Nesse sentido, cabe aos profissionais de saúde que compõem a ESF, em especial aos enfermeiros, realizar o diagnóstico situacional da comunidade escolar quanto à temática em estudo, planejar a educação em saúde, implementar e avaliar essa atividade de forma holística e com raciocínio crítico-reflexivo acerca da situação em que os estudantes e/ou professores encontram-se envolvidos. ${ }^{18}$

\section{Conclusão}

Os resultados desta pesquisa demonstraram que, por meio das intervenções educativas, os alunos obtiveram aprendizado significativo no ambiente escolar quanto ao desenvolvimento desses tipos de estratégias educativas. Além disso, este estudo pode contribuir com os gestores na compreensão da importância da inclusão da disciplina de primeiros socorros no currículo escolar desde o ensino fundamental.

Em estudos posteriores, pretende-se realizar tais estratégias educativas com um maior número de escolares e ampliar a realização das oficinas com professores do ensino fundamental, pois o ambiente escolar é propício para a ocorrência de acidentes e os professores são os primeiros a terem contato com a criança; porém, a maioria deles não tem conhecimento adequado para realizar o primeiro atendimento.

Este estudo apresentou como limitação o quantitativo de escolas e estudantes que participaram da pesquisa, a realização somente com estudantes do ensino fundamental e o pequeno número de encontros. Ainda, a elaboração do material didático contou com os conhecimentos dos professores, utilizando livros e manuais sobre a temática; foi, porém, proporcionado aos estudantes participarem de forma ativa no processo de abordagem do conteúdo e esclarecerem as dúvidas no processo. 


\section{Referências}

1. Vilaça L, Cardoso PR. Intoxicações na infância: panorama geral do perfil das intoxicações em diferentes países. Rev Méd Minas Gerais [Internet]. 2014 [acesso em 2018 out 14];24(1):21-5. Disponível em: http://rmmg.org/artigo/detalhes/597 doi:10.5935/2238-3182.20140012

2. Gonçalves AC, Echevarría-Guanilo ME, Gonçalves N, Rossi LA, Farina Júnior JA. Caracterização de pacientes atendidos em um serviço de queimados e atitudes no momento do acidente. Rev Eletrônica Enferm [Internet]. 2012 out-dez [acesso em 2018 set 24];14(4):866-72. Disponível em: http://fen.ufg.br/revista/v14/n4/pdf/v14n4a15.pdf doi:10.5216/ree.v14i4.15186

3. Costa CWA, Moura DL, Costa FLO, Melo RS, Moreira SR. Unidade didática de ensino dos primeiros socorros para escolares: efeitos do aprendizado. Pensar Prát [Internet]. 2015 abr-jun [acesso em 2018 out 10];(18)2:338-49. Disponível em: https://www.revistas.ufg.br/fef/article/view/30205 doi: 10.5216/rpp.v18i2.30205

4. Oliveira MR, Leonel ARA, Montezeli JH, Gastaldi AB, Martins EAP, Caveião C. Conception of undergraduate nursing students on the practice of health education on first aid. Rev RENE [Internet].2015 mar-abr [acesso em 2018 out 14];16(2):150-8. Disponível em: http://periodicos.ufc.br/rene/article/view/2696/2081 doi:10.15253/2175-6783.2015000200003

5. Patrício ACFA, Santos JS, Albuquerque KF, Alves KL, Duarte MCS, Pérez VLAB. Atendimento préhospitalar móvel: identificando agravos à saúde da pessoa idosa. Rev Pesqui Cuid Fundam [Internet]. 2016 abr-jun [acesso em 2018 nov 05];8(2):4223-30. Disponível em: http://seer.unirio.br/index.php/cuidadofundamental/article/view/4217 doi: https://doi.org/10.9789/2175-5361

6. Guimarães HP, Olivato GB, Pispico A. Ressuscitação cardíaca pré-hospitalar do pré-hospitalar à sala de emergência: minutos que salvam uma vida - suporte básico. Rev Soc Cardiol Estado de São Paulo [Internet]. 2018 [acesso em 2018 set 20];28(3):302-11. Disponível em: http://socesp.org.br/revista/assets/upload/revista/7626911271539115635pdfptRESSUSCITAÇÃO\%20CARD ÍACA\%20PRÉ-HOSPITALAR.\%20DO\%20PRÉ-

HOSPITALAR\%20À\%20SALA\%20DE\%20EMERGÊNCIA_REVISTA\%20SOCESP\%20V28\%20N3.pdf doi: http://dx.doi.org/10.29381/0103-8559/20182803302-11

7. Presidência da República (BR). Subchefia para Assuntos Jurídicos. Lei no 13.722, de 4 de outubro de 2018: torna obrigatória a capacitação em noções básicas de primeiros socorros de professores e funcionários de estabelecimentos de ensino públicos e privados de educação básica e de estabelecimentos de recreação infantil [Internet]. 2018 [acesso em 2019 abr 24]. Disponível em: http://www.planalto.gov.br/ccivil_03/_ato2015-2018/2018/lei/L13722.htm

8. Calandrim LF, Santos AB, Oliveira LR, Massaro LG, Vedovato CA, Boaventura AP. First aid at school: teacher and staff training. Rev RENE [Internet]. 2017 maio-jun [acesso em 2018 set 14];18(3):292-9. 
A escola como espaço para aprendizado sobre primeiros socorros | 14

Disponível em: http://periodicos.ufc.br/rene/article/view/20045/30696 doi: 10.15253/21756783.2017000300002

9. Pereira KC, Paulino JR, Saltarelli RMF, Carvalho AMP, Santos RB, Silveira TVL, et al. A construção de conhecimentos sobre prevenção de acidentes e primeiros socorros por parte do público leigo. Rev Enferm Cent-Oeste Min [Internet]. 2015 jan-abr [acesso em 2018 out 14];5(1):1478-85. Disponível em: http://www.seer.ufsj.edu.br/index.php/recom/article/viewFile/456/837

10. Mesquita TM, Albuquerque RS, Bonfim AMA, Sales MLH, Santana MCCP, Ferreira AMV. Educational resource to first aid education in the teaching-learning process for public school children. Rev Ciênc Plur [Internet]. 2017 [acesso em 2018 nov 08];3(1):35-50. Disponível em: http://pesquisa.bvsalud.org/portal/resource/pt/biblio-848592

11. Sönmez Y, Uskun E, Pehlivan A. Knowledge levels of pre-school teachers related with basic first-aid practices, Isparta sample. Türk Ped Arş [Internet]. 2014 [acesso em 2018 nov 10];49(3):238-46. Disponível em: https://www.journalagent.com/tpa/pdfs/TPA_49_3_238_246.pdf doi: http://doi.org/10.5152/tpa.2014.1581

12. Joseph N, Narayanan T, Bin Zakaria S, Nair AV, Belayutham L, Subramanian AM, et al. Awareness, attitudes and practices of first aid among school teachers in Mangalore, South India. J Prim Health Care [Internet]. 2015 [acesso em 2018 out 14];7(4):274-81. Disponível em: https://www.publish.csiro.au/hc/pdf/HC15274 doi: 10.1071/HC15274

13. Kawakame PMG, Miyadahira AMK. Assessment of the teaching-learning process in students of the health area: cardiopulmonary resuscitation maneuvers. Rev Esc Enferm USP [Internet]. 2015 [acesso em 2018 out 14];49(4):652-8. Disponível em: http://www.scielo.br/scielo.php?script=sci_arttext\&pid=S008062342015000400657 doi: 10.1590/S0080-623420150000400017

14. Martins AR. Educación para la salud en primeros auxilios dirigida al personal docente del ámbito escolar. Enferm Univ [Internet]. 2015 [acesso em 2018 set 14];12(2):88-92. Disponível em: https://www.sciencedirect.com/science/article/pii/S1665706315000056 doi: 10.1016/j.reu.2015.04.004

15. Ministério da Saúde (BR). Passo a Passo PSE: Programa de Saúde na Escola [Internet]. Brasília (DF): Ministério da Saúde; 2011 [acesso em 2020 jan 15]. Disponível em: http://189.28.128.100/dab/docs/legislacao/passo_a_passo_pse.pdf

16. Secretaria de Saúde do Distrito Federal (BR). Projeto Samuzinho [Internet]. Brasília (DF); 2018 [acesso em 2020 jan 15]. Disponível em: http://www.saude.df.gov.br/projeto-samuzinho/

17. Oliveira IS, Souza IP, Marques SM, Cruz AF. Knowledge of educators on prevention of accidents in childhood. Rev Enferm UFPE On Line [Internet]. 2014 [acesso em 2019 abr 25];8(2):279-85. Disponível em: 
15 | Grimaldi MRM, Gonçalves LMS, Melo ACOS, Melo FI, Aguiar ASC, Lima MMN

https://periodicos.ufpe.br/revistas/revistaenfermagem/article/view/9672 doi: 10.5205/reuol.4688-38583-1RV.0802201407

18. Galindo Neto NM, Carvalho GCN, Castro RCMB, Caetano JA, Santos ECB, Silva TM, et al. Teachers' experiences about first aid at school. Rev Bras Enferm [Internet]. 2018 [acesso em 2019 abr 25];71(Supl 4):1678-82. Disponível em: http://www.scielo.br/pdf/reben/v71s4/pt_0034-7167-reben-71-s4-1678.pdf doi: http://dx.doi.org/10.1590/0034-7167-2017-0715

\section{Autor correspondente}

Morgama Mara Nogueira Lima

E-mail: morgamamara@gmail.com

Endereço: Rua Florêncio Coelho Holanda, 550, casa 300. Bairro: Maraponga. Fortaleza, Ceará, Brasil. CEP: $60.711-220$

\section{Contribuições de Autoria}

\section{1 - Monaliza Ribeiro Mariano Grimaldi}

Concepção do projeto; condução da coleta e análise dos dados; e aprovação da versão final a ser publicada.

\section{2 - Luci Maira Silva Gonçalves}

Concepção do projeto; coleta e análise dos dados.

\section{3 - Ana Carolina de Oliveira Silva Melo}

Coleta, análise e interpretação dos dados.

\section{4 - Francisco Ivandi Melo}

Coleta, análise e interpretação dos dados.

\section{5 - Adriana Sousa Carvalho de Aguiar}

Revisão crítica relevante do conteúdo intelectual e aprovação da versão final a ser publicada.

\section{6 - Morgama Mara Nogueira Lima}

Revisão crítica relevante do conteúdo intelectual.

\section{Como citar este artigo}

Grimaldi MRM, Gonçalves LMS, Melo ACOS, Melo FI, Aguiar ASC, Lima MMN. A escola como espaço para aprendizado sobre primeiros socorros. Rev. Enferm. UFSM. 2020 [Acesso em: Anos Mês Dia]; vol.10 e: 1-15. DOI:https://doi.org/10.5902/2179769236176 